of prognosis, and in some cases, more specific treatment. Screening for rare CNVs is a valuable routine diagnostic workup in patients with unclassified epilepsies and complex phenotypes; 88 rare CNVs were discovered in 71 of 222 patients (31.9\%) [1]. A chromosomal microarray (CMA) screen of 215 patients with a broad range of neurological phenotypes of unknown etiology found 30 (14\%) were abnormal; phenotypes included infantile spasms, other epilepsies, and cortical malformations [2].

CMA is recommended especially in unclassified epilepsy patients with dysmorphic features, developmental delay, autistic spectrum disorder, family history of epilepsy, or parental consanguinity. More directed genetic testing for specific epilepsy genes or genetic neurological syndromes is indicated for patients with a characteristic phenotype [3].

\title{
References.
}

1. Helbig I, et al. Eur J Hum Genet. 2014 Jul;22(7):896-901.

2. Howell KB, et al. J Paediatr Child Health. 2013 Sep;49(9):716-24.

3. Millichap JG. Neurological Syndromes : A Clinical Guide to Symptoms and Diagnosis. New York: Springer; 2013:279.

\section{COMPARISON OF 5- AND 30-MINUTE STATUS EPILEPTICUS}

Investigators at Boston Children's Hospital and other centers compared the characteristics of patients with status epilepticus (SE) lasting 5-29 min with those with SE lasting $>30 \mathrm{~min}$. Of a total 445 patients with a median age at SE of $5.5(2.8-10.5)$ years, $296(66.5 \%)$ had SE lasting 5-29 min, and in 149 (33.5\%) SE lasted $>30 \mathrm{~min}$. Patients with SE $>30$ min were younger than those with SE 5-29 min at time of seizure onset (median 1 vs 2.1 years, $\mathrm{p}=0.0007$ ). $\mathrm{SE}$ as the first seizure presentation was more frequent in patients with $\mathrm{SE}>30 \mathrm{~min}(24.2 \%$ vs $12.2 \%, \mathrm{p}=0.002)$. MRI abnormalities tended to be more frequent in patients with $\mathrm{SE}>30 \mathrm{~min}(70.5 \%$ vs $57.1 \%, \mathrm{p}=0.061)$. Shorter vs longer SE cases showed no differences in seizure frequency, seizure types (febrile vs nonfebrile), developmental delay, and EEG abnormalities at baseline. At a median follow-up of 3.7 years after SE, 21 patients $(4.7 \%)$ had died (median time of 3.1 years after SE), mostly unrelated to epilepsy. The odds of dying showed an increase of 0.005 per min of SE duration. SE thresholds of either 5 or 30 min identify groups of patient with similar electroclinical characteristics. (Fernandez IS, Vendrame M, Kapur K, et al. Comparison of pediatric patients with status epilepticus lasting 5-29 min versus $>30$ min. Epilepsy Behav 2014 Jun 17;37C:1-6).

COMMENTARY. Longer SE duration ( $>30 \mathrm{~min}$ ) probably reflects a more severe underlying cause for SE, but patients with shorter (5-29 min) SE have comparable electroclinical characteristics to those with longer SE duration. Since seizures are more difficult to treat and carry a poorer outcome the longer they last [1][2], seizures lasting 5 min or longer are treated as aggressively as those $>30$ min duration. Seizures (5-29 min) are considered as "impending" SE and those $>30$ min as "established" SE.

\section{References.}

1. Eriksson K, et al. Neurology. 2005 Oct 25;65(8):1316-8.

2. Seinfeld S, et al. Epilepsia. 2014 Mar;55(3):388-95. 